\title{
Subject Index to Volume 17, 2004
}

Abscess, tuboovarian, fatal pneumococcal sepsis from, 17(1):68-70

Adolescent(s), with ADHD, on-road driving by, controlled-release methylphenidate and, 17(4):235239

Advertising, direct-to-consumer, public perceptions of, 17(1):6-18

Alcohol, carbohydrate-deficient transferrin as biomarker of, in diabetic/hypertensive patients, 17(4): 247-255

Alcohol intoxication, nontraumatic rhabdomyolysis with, 17(1):54-58

Angina, Prinzmetal, multiple sclerosis with, 17(1):71-73

Antibiotics, for common cold, physician specialty and, 17(3):196-200

Apnea, obstructive sleep, in pregnancy, 17(4):292-294

Arctic Circle, racial prejudice in, 17(1): $78-79$

Asthma

in Medicaid-insured children, emergency room use by, 17(2):96-100

practical clinical trial for, conducted in primary care, 17(3):190-195

Attention-deficit/hyperactivity disorder (ADHD), in adolescent, on-road driving by, controlled-release methylphenidate and, 17(4):235239

Back pain, management in family practice, 17:S23-S31

Breast cancer, diagnostic rate in postmenopausal women with selfreported breast symptoms, 17(6): 408-415

Breast-feeding, early termination of, parental mental health and, 17(3):173-183

Bursitis, subacromial septic, from corticosteroid injection, 17(4):299-302

California, Spanish-speaking physicians in, access to, 17(3):165-172

Cancer

of breast, in postmenopausal women, self-reported breast symptoms and diagnostic rate in, 17(6):408-415

of skin, overcoming obstacles to examination and prevention counseling, 17(6):416-423

Cancer pain

high-dose fentanyl patch for, 17(5) $388-390$

management by family physician, 17:S48-S56

Carbohydrate-deficient transferrin, as alcohol biomarker in diabetic/ hypertensive patients, 17(4):247255

Cardiovascular disease, in women, dyslipidemia and, 17(6):424-437
Cerebral venous thrombosis, acute headache caused by, 17(4):295298

Cerebrospinal fluid neutrophilic pleocytosis, in hospitalized West Nile virus patients, 17(6):470-472

Chest pain, panic disorder and coronary artery disease in, 17(2):114-126

Childbirth, assisted, avoiding with knotted bedsheet, 17(5):394-395

Children

Medicaid-insured, with asthma, emergency room use by, 17(2): 96-100

well-child visit noncompliance in, parental health and demographics in, 17(5):324-331

Clinical practice guidelines

compliance with, 17(6):474-475

physician opinions and attitudes about, 17(2):150-157

Clinical trial, of asthma, conducted in primary care, 17(3):190-195

Colonoscopy, in rural communities, by family physician, safety and effectiveness of, 17(5):353-358

Colorado, primary care by specialists in, 17(2):81-90

Community family practice

physician-older patient interaction in 17(1):19-25

public health and, 17(3):207-211

Congestive heart failure, rofecoxib-induced, 17(2):131-135

Continuity of care, patient satisfaction and, in family practice clinic, 17(5):341-346

Coronary artery bypass surgery, Dressler syndrome after, 17(3): 230-232

Coronary artery disease, panic disorder and, 17(2):114-126

Correspondence

challenges for family medicine and for family physicians, 17(6):474

compliance with clinical guidelines and the law of thirds, 17(6):475

impact of clinical practice guidelines should not be overestimated, 17(6):474-475

Corticosteroid injection, subacromial septic bursitis from, 17(4):299302

Cough, pancreatic adenocarcinoma and, 17(1):48-53

Counseling, on skin cancer prevention, 17(6):416-423

Cyclooxygenase inhibitors, congestive heart failure induced by, 17(2): 131-135

Deep-breath test, white-coat hypertension and, 17(3):184-189

Demographics, parental, well-child visit noncompliance and, 17(5):324331
Diabetes mellitus

borderline, peripheral neuropathy and, 17(2):127-130

carbohydrate-deficient transferrin as alcohol biomarker in, 17(4):247255

Diabetes mellitus type 2, lipid management in, 17(2):101-107

Diapers, gel-based, urine culture collected from, 17(2):91-5

Dressler syndrome, after coronary artery bypass surgery, 17(3):230232

Driving

by ADHD adolescents, controlled release methylphenidate improvement of, 17(4):235-239

by older persons, physician assessment of, 17(1):38-43

Drug prescribing, multitiered incentivebased formularies in, 17(6):401407

Dyslipidemia, in women, cardiovascular disease and, 17(6):424-437

Dysmenorrhea

pelvic pain from, management in family practice, 17:S43-S47

primary, guaifenesin for, 17(4):240_ 246

Ecuador, obstetrics advanced life support in, 17(4):276-282

Editorial, how long does it take to become a competent family physician?, 17(5):391

Emergency room use, by Medicaidinsured children with asthma 17(2):96-100

Endometriosis, pelvic pain from, management in family practice, 17 : S43-S47

Endoscopy, acute methemoglobinemia after, 17(3):227-229

Esophagogastroduodenoscopy, officebased ultrathin, 17(6):438-442

Evidence-based medicine

obesity in adults, diagnosis and treatment of, 17(5):359-369

office radiographs, second reading of, 17(4):256-263

Expiration dates, on medicine containers, location, content, and legibility of, 17(5):395-397

Exposure assessment, assessment of neuropsychiatric symptom relief and, 17(2):136-141

Factor $\mathrm{V}$ Leiden mutation, pregnancy and, 17(4):306-308

Family practice clinic, continuity of care and patient satisfaction in, 17(5):341-346

Fentanyl patch, high-dose, for cancer pain, 17(5):388-390

Fever, in respiratory infection, 17(1): 32-37 
Food and Drug Administration (FDA), approval process of, tensions in, 17(2):142-149

Formularies, multitiered incentivebased, 17(6):401-407

Genital dysplasia, in women with HIV infection, 17(2):108-113

Glucose tolerance, impaired, peripheral neuropathy and, 17(2):127-130

Guaifenesin, for primary dysmenorrhea, 17(4):240-246

Headache, acute, caused by cerebral venous thrombosis, 17(4):295298

Health care costs, very low literacy skills and, 17(1):44-47

Health care policy, tensions in FDA approval process, 17(2):142-149

Health care system, care providers and settings for Medicare beneficiaries in, 17(5):384-387

Health Insurance Portability and Accountability Act (HIPAA) Privacy Rule, for practice-based researchers, 17(6):461-465

Heart attack, neck pain mimicking, 17(1):74-77

Herpes simplex type 2 virus infection, meningitis from, 17(4):303-305

Human immunodeficiency virus infection, in women, genital dysplasia in, $17(2): 108-113$

Human papillomavirus infection, knowledge and behaviors related to, 17(1):26-31

Hypertension

carbohydrate-deficient transferrin as alcohol biomarker in, 17(4):247255

white-coat, deep-breath test and, 17(3):184-189

\section{Infection}

herpes simplex virus type 2, meningitis from, 17(4):303-305

$\mathrm{HIV}$, in women, genital dysplasia in, 17(2):108-113

human papillomavirus, knowledge and behaviors related to, 17(1): 26-31

respiratory

antibiotics for, physician specialty and, 17(3):196-200

febrile, 17(1):32-37

Influenza

diagnosis of, medical history and physical examination in, 17(1): $1-5$

vaccination against, in pregnancy, 17(4):287-291

Insomnia, primary, treatment of, 17(3): 212-219

Internal medicine specialists, and antibiotic prescribing for common cold, 17(3):196-200

Intimate partner violence, physician perceptions of, 17(5):332-340
Joint pain, management in family practice, 17:S32-S42

Law of thirds, 17(6):475

Life support, advanced obstetric, in Ecuador, 17(4):276-282

Lipid management, in diabetes type 2, 17(2):101-107

Literacy skills, very low, health care costs and, 17(1):44-47

Medicaid recipients

pediatric asthma patients as, emergency room use by, 17(2):96100

very low literacy skills in, health care costs and, 17(1):44-47

Medical history, in influenza diagnosis, 17(1):1-5

Medical literature, grading evidence in, SORT in, 17(1):59-67

Medicare beneficiaries, care providers and settings for, 17(5):384-387

Medicine containers, expiration dates on, location, content, and legibility of, 17(5):395-397

Meningitis, Mollaret, 17(4):303-305

Mental health, parental, breast-feeding early termination and, 17(3): 173-183

Metapneumovirus, human, 17(6):466469

Methemoglobinemia, acute, after endoscopy, 17(3):227-229

Methicillin-resistant Staphylococcus aureus, misdiagnosed as spider bite, 17(3):220-226

Methylphenidate, controlled release, and attention during on-road driving by ADHD adolescents, 17(4):235-239

Mollaret meningitis, 17(4):303-305

Multiple sclerosis, Prinzmetal angina with, 17(1):71-73

Neck pain

heart attack mimicked by, 17(1):74-77

management in family practice, 17 : S13-S22

Neuropathy

peripheral, in borderline diabetes, 17(2):127-130

peripheral sensory, in older patients, prevalence, predictors, and consequences of, 17(5):309-318

Neuropsychiatric symptoms, from occupational hazardous chemical exposure, 17(2):136-141

Neutrophilic pleocytosis, in cerebrospinal fluid of hospitalized West Nile virus patients, 17(6):470 472

Nonsteroidal anti-inflammatory drugs (NSAIDs), congestive heart failure induced by, 17(2):131-135

Nutrition labels, on snack food packages, patient understanding and use of, 17(5):319-323
Obesity, in adults, diagnosis and treatment of, 17(5):359-369

Obstetrics, advanced life support in, in Ecuador, 17(4):276-282

Obstructive sleep apnea, in pregnancy, 17(4):292-294

Occupational hazardous chemical exposure, assessment of, neuropsychiatric symptom relief and, 17(2): 136-141

Office radiographs, second reading of, 17(4):256-263

Office ultrathin esophagogastroduodenoscopy, 17(6):438-442

Older patients

deciding to change primary care physicians, when and why, 17(6): 453-460

driving ability of, physician assessment of, 17(1):38-43

peripheral sensory neuropathy in, prevalence, predictors, and consequences of, 17(5):309-318

physician interaction with, in community family practice, 17(1): $19-25$

Pain

in back, 17:S23-S31

cancer-related

in adult, 17:S48-S56

high-dose fentanyl patch for, 17(5): 388-390

in joints, 17:S32-S42

management by family physician, 17:S1-S12

in neck, 17:S13-S22

in pelvis, from dysmenorrhea or endometriosis, 17:S43-S47

Pancreatic adenocarcinoma, cough and, 17(1):48-53

Panic disorder, coronary artery disease and, 17(2):114-126

Papanicolaou smear, sigmoidoscope for, 17(3):233

Parent(s)

health and demographics of, wellchild visit noncompliance and, 17(5):324-331

mental health of, breast-feeding early termination and, 17(3):173-183

Patient satisfaction, continuity of care and, in family practice clinic, 17(5):341-346

Pelvic pain, from dysmenorrhea or endometriosis, management in family practice, 17:S43-S47

Peripheral sensory neuropathy, in older patients, prevalence, predictors, and consequences of, 17(5):309318

Physical examination, in influenza diagnosis, 17(1):1-5

Physician(s)

family

colonoscopy by, safety and effectiveness of, 17(5):353-358

practice-oriented and researchoriented, 17(6):474

family practice residents perspectives on length and content of training, 17(5):377-383, 17(5):391 
healing by patients, 17(2):158-159

intimate partner violence perceptions of, 17(5):332-340

older driver assessment by, 17(1): $38-43$

opinions and attitudes about clinical practice guidelines, 17(2):150 157

primary care, older patients deciding to change, when and why, 17(6): 453-460

Spanish-speaking, access to, in California, 17(3):165-172

specialists, providing primary care services in Colorado, 17(2):81-90

Physician-patient relationship

direct-to-consumer advertising effect on, public perceptions of, 17(1): $6-18$

older patients in

in community family practice, 17(1):19-25

who decide to change physicians, 17(6):453-460

Pneumococcal sepsis, fatal, from tuboovarian abscess, 17(1):68-70

Postmenopausal women, self-reported breast symptoms in, breast cancer diagnostic rate in, 17(6):408-415

Pregnancy

Factor V Leiden mutation and, 17(4): 306-308

influenza vaccination in, 17(4):287-291

obstructive sleep apnea in, 17(4):292294

smoking cessation in, postpartum relapse prevention strategies after, 17(4):264-275

Primary care

addressing spiritual concerns in, 17(3):201-206

antibiotic use for common cold in, 17(3):196-200

asthma clinical trial conducted in, 17(3):190-195

community-oriented, public health and, 17(3):207-211

office-based ultrathin esophagogastroduodenoscopy in, 17(6):438442

self-reported health status in, racial disparities in, 17(6):443-452

by specialists, in Colorado, 17(2): $81-90$

Prinzmetal angina, multiple sclerosis with, 17(1):71-73

Privacy rule, of HIPAA, for practicebased researchers, 17(6):461-465

Probability, communicating with patients about it, 17(6):473

Public health, family medicine and, 17(3):207-211

Racial disparities, in self-reported health status, in primary care, 17(6):443-452

Racial prejudice, in Arctic community, 17(1):78-79

Radiographs, in office, second reading of, 17(4):256-263
Rare conditions, in family practice, 17(4):283-286

Reflections in family practice

adopted, 17(2):158-159

welcome to the Arctic Circle, 17(1) $78-79$

Religion, in health outcome, 17(5):370-

Renal failure, acute, from nontraumatic rhabdomyolysis, with alcohol intoxication, 17(1):54-58

Research letters

numeracy and medicine: key family physician attitudes about communicating probability with patients, 17(6):473

using a bed sheet to avoid an assisted delivery, 17(5):394-395

variable location, content, and legibility of expiration dates on medicine containers, 17(5):395-397

Researchers, practice-oriented, 17(6):474

HIPAA Privacy Rule for, 17(6):461465

Respiratory infection antibiotics for, physician specialty and, 17(3):196-200

febrile, 17(1):32-37

Respiratory tract pathogen, human metapneumovirus as, 17(6):466469

Review articles, grading evidence in, SORT in, 17(1):59-67

Rhabdomyolysis, nontraumatic, with alcohol intoxication, 17(1):54-58

Rofecoxib, congestive heart failure induced by, 17(2):131-135

Rural communities, colonoscopy by family physician in, safety and effectiveness of, 17(5):353-358

Self-reported breast symptoms, by postmenopausal women, breast cancer rate in, 17(6):408-415

Self-reported heath status, in primary care, racial disparities in, 17(6): $443-452$

Sensory neuropathy, peripheral, in older patients, prevalence, predictors, and consequences of, 17(5):309-318

Sepsis, pneumococcal fatal, from tuboovarian abscess, 17(1):68-70

Septic bursitis, subacromial, from corticosteroid injection, 17(4):299302

Sigmoidoscope, for Papanicolaou smear, 17(3):233

Skin cancer, overcoming obstacles to examination and prevention counseling, 17(6):416-423

Sleep disorders

obstructive sleep apnea as, in pregnancy, 17(4):292-294

primary insomnia as, treatment of, 17(3):212-219

Smoking cessation, in pregnancy, postpartum relapse prevention strategies after, 17(4):264-275

Snack food package nutrition labels, patient understanding and use of, 17(5):319-323
Spanish-speaking physicians, access to, in California, 17(3):165-172

Special communications

addressing spiritual concerns in family medicine: a team approach, 17(3):201-206

family physicians' opinions and attitudes about clinical practice guidelines, 17(2):150-157

strength of recommendation taxonomy (SORT): patient-centered approach to grading evidence in medical literature, 17(1):59-67

zebras on the commons: rare conditions in family practice, 17(4) 283-286

Specialist(s), and antibiotic prescribing for common cold, 17(3):196-200

Specialists, providing primary care services in Colorado, 17(2):81-90

Spider bite

brown recluse, management of, 17(5):347-352

methicillin-resistant Staphylococcus aureus mimicking, 17(3):220-226

Spiritual concerns, addressing in family medicine, 17(3):201-206

Spirituality, in health outcome, 17(5): 370-376

Staphylococcus aureus, methicillinresistant, misdiagnosed as spider bite, 17(3):220-226

Strength of recommendation taxonomy (SORT), 17(1):59-67

Thrombosis, cerebral venous, acute headache caused by, 17(4):295298

Training in family practice, length and content of, 17(5):377-383, 17(5): 391

Transferrin, carbohydrate-deficient, as alcohol biomarker in diabetic/ hypertensive patients, 17(4):247255

Tuboovarian abscess, fatal pneumococcal sepsis from, 17(1):68-70

Urine, collection for culture, from gelbased diapers, 17(2):91-95

Vaccination, influenza, in pregnancy, 17(4):287-291

Violence, from intimate partner, physician perceptions of, 17(5):332-340

Well-child visits, noncompliance with, parent health and demographics in, 17(5):324-331

West Nile virus, cerebrospinal fluid neutrophilic pleocytosis in, 17(6):470-472

White-coat hypertension, deep-breath test and, 17(3):184-189

Women

cardiovascular disease in, dyslipidemia and, 17(6):424-437

postmenopausal, self-reported breast symptoms and breast cancer diagnostic rate in, 17(6):408-415 